OPEN ACCESS

Edited by:

Gaetano Gallo,

University of Catanzaro, Italy

Reviewed by:

Marco Frascio,

University of Genoa, Italy

Weidong Tong,

Army Medical University, China

*Correspondence:

Jonas Herzberg

jonas.herzberg@

krankenhaus-reinbek.de

tThese authors have contributed equally to this work and share last authorship

Specialty section:

This article was submitted to Visceral Surgery,

a section of the journal

Frontiers in Surgery

Received: 04 October 2021 Accepted: 07 December 2021

Published: 07 January 2022

Citation:

Herzberg J, Khadem S, Begemann V,

Strate T, Honarpisheh $\mathrm{H}$ and Guraya SY (2022) Quality of Life in Patients With Rectal Resections and End-to-End Primary Anastomosis Using a Standardized Perioperative Pathway. Front. Surg. 8:789251 doi: $10.3389 /$ fsurg.2021.789251

\section{Quality of Life in Patients With Rectal Resections and End-to-End Primary Anastomosis Using a Standardized Perioperative Pathway}

\author{
Jonas Herzberg ${ }^{1 *}$, Shahram Khadem ${ }^{1}$, Valentin Begemann ${ }^{1}$, Tim Strate ${ }^{1}$, \\ Human Honarpisheh ${ }^{1 \dagger}$ and Salman Yousuf Guraya ${ }^{2 \dagger}$ \\ ${ }^{1}$ Department of Surgery - Krankenhaus Reinbek St. Adolf-Stift, Reinbek, Germany, ${ }^{2}$ Clinical Sciences Department, College \\ of Medicine, University of Sharjah, Sharjah, United Arab Emirates
}

Objectives: Lower rectal resection is associated with a high rate of postoperative complications and, therefore, adversely impacts the postoperative health-related quality of life (QoL). Though sporadically practiced in different centers, there is no standard perioperative protocol for the management of patients with rectal growths. The aim of this analysis is to evaluate the patient-reported outcomes after low rectal resections followed by an end-to-end-reconstruction and temporary covering ileostomy using a multidisciplinary fail-safe-concept.

Methods: Between 2015 and 2020, we evaluated patient reported outcomes after open and laparoscopic rectal resections with end-to-end reconstruction with a primary straight anastomosis using a standardized perioperative pathway All patients with stoma were excluded from the study. The data for the QoL of patients was collected using the established Low Anterior Resection Syndrome (LARS)-score and the EORTC-C30 and CR-29 questionnaires at a single postoperative timepoint.

Results: We recruited 78 stoma-free patients for this analysis. Of 78 patients included in the study, $87.2 \%$ were operated laparoscopically and the mean global health status was 67.95 points, while a major LARS was detected in 48 (61.5\%) patients. No anastomotic leakage (AL) occurred within the study cohort. There was no significant change in the LARS-score or the global health status depending on the follow-up-period.

Conclusion: This study shows that good QoL and functional outcomes with no AL are achievable following end-to-end straight anastomosis using a standardized perioperative surgical fail-safe protocol procedure.

Keywords: rectal resection, PROM (patient reported outcome measures), quality of life, colorectal surgery, anastomosis

\section{INTRODUCTION}

Colorectal cancer is the third leading cancer worldwide and the second leading cause of cancer-related deaths with an estimated 1.8 million new cases per year and 881,000 deaths worldwide $(1,2)$. Surgical therapy remains the gold standard for rectal growths and the outcomes of surgical treatment for rectal pathologies primarily depend on the location 
and stage of the tumor, the perioperative surgical protocol and the surgical technique (3). The most reported adverse mid-termconsequence of low anterior resection (LAR) is a deranged bowel function, often referred as "low anterior resection syndrome" (LARS) (4). The manifestations of LARS are far ranging; fecal incontinence, urgency, evacuatory and sexual dysfunctions, abnormal bowel frequency. As evident, LARS carry a direct impact on the quality of life (QoL) after rectal surgery (5). QoL, the individual's state of wellbeing, is deeply influenced by illness and treatment, especially in cancer patients (6).

In the recent years, the health-related quality of life (HRQOL) has been recognized as a mandatory requirement for the approval process of new anticancer drugs by the European Medicines Agency (7). Additionally, during the routine management of patients with a range of elements, the HRQOL has been embedded as a part of the patient-reported outcomes. LAR with primary anastomosis carries a high risk for adverse postoperative patient-reported outcomes due to a rise in postoperative complications such as anastomotic leakage (AL), sepsis and delayed bowel functions (8). Moreover, sphincter-preserving rectal surgery often leads to autonomic nerve damage with its associated functional disorders. Following LAR for the rectal cancer, defecation disorders have been reported in $41 \%$ (9), sexual dysfuction in 64\% (10) and urinary dysfunctions in 50\% patients (10). Such alarming rates of complications following LAR adversely affect the patient's psychosocial health status and the HRQOL.

Different tools for the assessment of patient-reported outcomes and HRQOL for rectal resections have been tested and validated. For a short-term evaluation, a popular instrument is the time-tested 5-item LARS score, which includes questions for incontinence for flatus or stool, the frequency of bowel movement, incomplete defecation, or urgency (11-13). This simple tool, available in different languages (13-15), focuses on the postoperative defecation disorders following rectal resections and correlates well with other QoL questionnaires (16).

In addition to the LARS questionnaire, the European Organization for Research and Treatment of Cancer (EORTC) has developed a well-evaluated 30-item core questionnaire (QLQ-C30), which investigates the general QoL with additional procedure-related instruments such as the questionnaire for the colorectal cancer (QLQ-C29) (17).

A range of remedial steps have been taken to prevent the dreadful long-term functional complications following rectal resections for cancerous growths. To reduce the rate of autonomic nerve complications, the intra-operative autonomic nerve preservation has been successfully established (18-21). This mandates the use of laparoscopic or robotic surgery in high-volume centers by experienced colorectal surgeons. The reservoir functions of the rectum is lost following the resection for the rectum along with its ampulla (22). In order to prevent a high stool frequency, the bowel reconstruction could be performed using a J-pouch (23), a coloplasty (24) or side-toend-reconstruction $(22,25)$. This surgical step might reduce the frequency of defecation; however, the placement of sutures or stapling lines can possibly lead to an increased leakage rate. Following these beneficial observations in the literature, the
German guidelines for colorectal cancer surgery recommend the use of a reservoir building reconstruction such as pouch or end-to-side-reconstruction, wherever possible (26).

Despite an escalating rise in the rates of complications and poor QoL after rectal surgery, unfortunately there is no standard peri-operative management protocol that can mitigate these risks. Though literature has shown the QoL related outcomes of patients following rectal surgery using cross-sectional study designs, there is a limited data about the reference population or pretreatment guidelines $(27,28)$.

The aim of this study is to evaluate the patient-reported outcomes after low rectal resections and end-to-endreconstruction for benign and malignant rectal lesions using a multidisciplinary fail-safe-protocol. We used the EORTC-C30, C29 questionnaires and the LARS-score for the assessment of the QoL after rectal resections, which provide an insight into the efficacy and safety profile of the fail-safe peri-operative protocol.

\section{MATERIALS AND METHODS}

\section{Study Design}

In this study, we prospectively included all patients who underwent open or laparoscopic rectal resections with end-toend anastomosis at Reinbek St. Adolf-Stift Hospital Germany to a colorectal surgical database. Between January 2015 and December 2020, patients with a tumor localized $\leq 8 \mathrm{~cm}$ from the anal verge were treated by rectal resections and end-to-end primary anastomosis. The hallmark of our management plan was the multi-modal fail-safe protocol, which included a standard

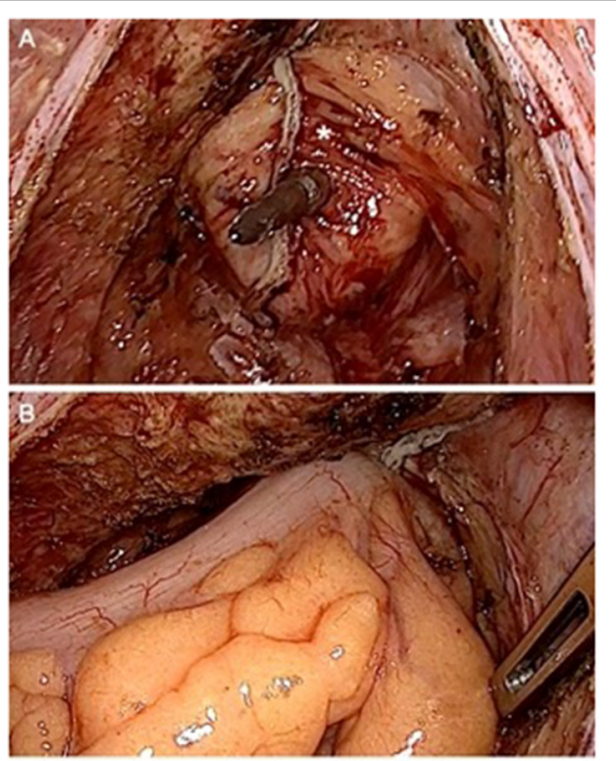

FIGURE 1 | Performing an end-to-end reconstruction after low rectal resection. (A) Rectal stump ( $\left.{ }^{\star}\right)$ still covered with fatty tissue to ensure perfusion with the spine of the stapler is piercing near the previous stapling line. (B) Compression after joining both ends to flatten fatty tissue before releasing the stapling device. 
surgical technique for tension-free anastomosis was adapted in the fail-safe approach. All patients with rectal resections and primary end-to-end-anastomosis had an ileostomy, which were closed after the completion of adjuvant therapy soonest 6 weeks after the primary procedure. Patients who still had ileostomies at the time of conducting this study were excluded from the cohort. The patients' median follow-up period was 1 year.

After obtaining the ethical approval, the patients' medical records were extracted from the prospective clinical database according to the established inclusion criteria. Later, all recruited patients were invited to participate in this research. All patients gave their written informed consent to participate in this study. The EORTC-C30, C29 questionnaires and the LARS-scoring tool were posted to all patients by registered post at a single timepoint. The data presented in this study are reported in concordance with the STrengthening the Reporting of OBservational studies in Epidemiology (STROBE) criteria (29). This trail was registered in the German Clinical Trial Register (DRKS00022492, date of registration: 10/20/2020).

\section{Perioperative Fail-Safe Protocol}

All patients suspected with rectal cancers were staged according to the German guidelines for colorectal cancer (26). Depending on the preoperative staging and according to the decision of the interdisciplinary tumor board, patients were treated by neoadjuvant radiotherapy, chemoradiotherapy or primary surgery. Patients with benign rectal lesions were not discussed during the interdisciplinary tumor board meetings. In case of severe diverticulitis, extended resections were performed.

All patients were treated according to the fail-safe-protocol with a preoperative mechanical bowel preparation using $2 \mathrm{l}$ of

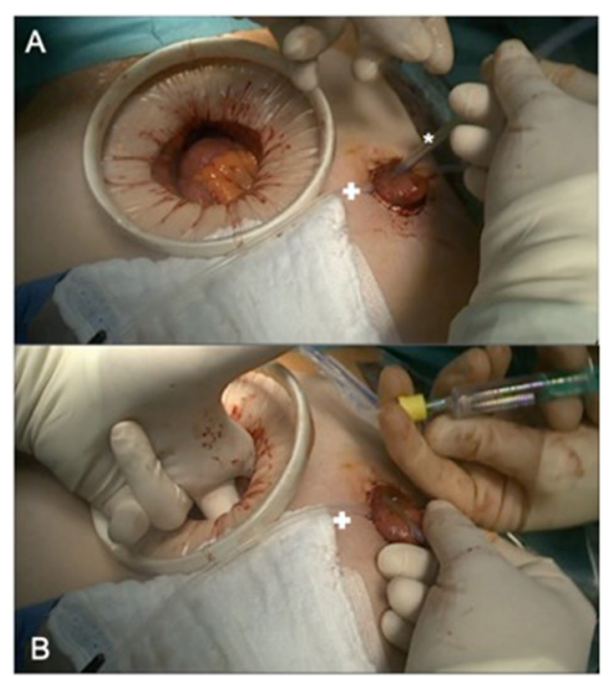

FIGURE 2 | Intraoperative colonic irrigation via ileostomy. (A) Placing the catheter $\left(^{\star}\right)$ in the efferent loop (+ marking a loop, fixing the diverting stoma until fixation is completed. (B) Blocking the catheter under manual controll before starting the antegrade colonic irrigation.

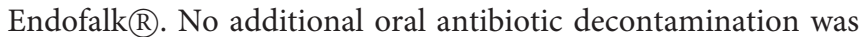
deemed necessary. In case of primary open procedures, epidural anesthesia was established. A single-shot-antibiotic was given intravenously perioperatively using $500 \mathrm{mg}$ metronidazole and $1500 \mathrm{mg}$ cefuroxime. After performing an end-to-end stapling anastomosis (Figure 1), a drainage was placed in the pelvis near the anastomosis and a diverting ileostomy was performed. In addition, an on-table-lavage via the efferent loop of the ileostomy was used to reduce the fecal load near the anastomosis (Figure 2). Three days after surgery, an endoscopic evaluation of the anastomosis was routinely performed. The diverting ileostomy was reversed after the completion of adjuvant therapy, if needed, at least 6 weeks after surgery and after performing colonoscopy for the evidence of intact anastomosis.

Postoperative complications were graded in accordance to the established Dindo-Clavien grading system, where all

TABLE 1 | Patient characteristics according to LARS/Major LARS.

\begin{tabular}{|c|c|c|c|c|}
\hline & $\begin{array}{c}\text { Total } \\
(n=78)\end{array}$ & $\begin{array}{c}\text { No/minor } \\
\text { LARS } \\
(n=30)\end{array}$ & $\begin{array}{l}\text { Major LARS } \\
\quad(n=48)\end{array}$ & $P$ \\
\hline $\begin{array}{l}\text { Age [years] } \\
(\text { mean } \pm S D)\end{array}$ & $65.64 \pm 12.24$ & $65.60 \pm 12.71$ & $65.67 \pm 12.08$ & $N^{c}$ \\
\hline $\mathrm{BMI}($ mean $\pm \mathrm{SD})$ & $27.51 \pm 8.89$ & $25.94 \pm 3.83$ & $28.49 \pm 10.86$ & $N^{c}$ \\
\hline \multicolumn{5}{|l|}{ Sex, n (\%) } \\
\hline Male & $45(57.7)$ & $17(56.7)$ & 28 (58.3) & $N^{b}$ \\
\hline Female & 33 (42.3) & $13(43.3)$ & $20(41.7)$ & $N S^{b}$ \\
\hline \multicolumn{5}{|l|}{ ASA, n (\%) } \\
\hline 1 & $4(5.1)$ & $3(10.0)$ & $1(2.1)$ & $N S^{b}$ \\
\hline$\|$ & $54(69.2)$ & $20(66.7)$ & $34(70.8)$ & $N S^{b}$ \\
\hline III & $20(25.6)$ & 7 (23.3) & $13(27.1)$ & $N S^{b}$ \\
\hline \multicolumn{5}{|l|}{ Procedure, n (\%) } \\
\hline $\begin{array}{l}\text { Laparoscopic, n } \\
\text { (\%) }\end{array}$ & 68 (87.2) & 25 (83.3) & 43 (89.6) & $N S^{b}$ \\
\hline Open & 8 (10.3) & 4 (13.3) & $4(8.3)$ & $N S^{b}$ \\
\hline Conversion & $2(2.6)$ & $1(3.3)$ & $1(2.1)$ & $N S^{b}$ \\
\hline $\begin{array}{l}\text { Length of surgery } \\
\text { [min] (mean } \pm \text { SD) }\end{array}$ & $263.36 \pm 79.45$ & $228.50 \pm 71.37$ & $285.15 \pm 77.05$ & 0.001 \\
\hline $\begin{array}{l}\text { Time to follow-up } \\
\text { [months] (mean } \pm \\
\text { SD) }\end{array}$ & $19.50 \pm 16.86$ & $22.07 \pm 17.77$ & $17.90 \pm 16.24$ & $N^{c}$ \\
\hline $\begin{array}{l}\text { Global Health status } \\
\text { (mean } \pm \mathrm{SD})\end{array}$ & $67.95 \pm 20.37$ & $75.83 \pm 18.49$ & $63.02 \pm 20.11$ & $0.003^{c}$ \\
\hline $\begin{array}{l}\text { Major complication } \\
(\mathrm{DC}>3 \mathrm{~b}), \mathrm{n}(\%)\end{array}$ & $4(5.1)$ & $4(5.1)$ & $0(0)$ & $0.019^{b}$ \\
\hline \multicolumn{5}{|l|}{ Dignity, n (\%) } \\
\hline Benign & $16(20.5)$ & $8(26.7)$ & 8 (16.7) & $N S^{b}$ \\
\hline \multirow[t]{2}{*}{ Malign } & 62 (79.5) & 22 (73.3) & 40 (83.3) & $N S^{b}$ \\
\hline & $n=62$ & $n=22$ & $n=40$ & \\
\hline $\mathrm{N}+(\%)^{\mathrm{a}}$ & $24(38.70)^{a}$ & $9(40.9)$ & $15(37.5)$ & $N^{b}$ \\
\hline T3/4 (\%) & $29(46.8)^{a}$ & $10(45.5)$ & $19(47.5)$ & $\mathrm{NS}^{\mathrm{b}}$ \\
\hline $\mathrm{RO}, \mathrm{n}(\%)^{\mathrm{a}}$ & $61(98.4)^{a}$ & $22(100.0)$ & 39 (97.5) & $N S^{b}$ \\
\hline
\end{tabular}

DC, Dindo-Clavien classification.

a Including only cases with malignancy $(n=62)$.

${ }^{b}$ Fisher exact test.

${ }^{c}$ Man-Whitney-U-test. 
complications graded $3 \mathrm{~b}$ and above are considered as major complications (30).

\section{Patient Reported Outcomes}

The EORTC-C30, C29 questionnaires and the LARS-scoring tool were used to measure the patient-reported outcomes and postoperative functional results in colorectal surgery.

\section{LARS Score}

The LARS score is a well-established simple scoring tool for the evaluation of bowel function after rectal resections. This tool assesses postoperative incontinence for flatus and liquid stool, frequency of bowel movement, incomplete defecation and urgency $(13,31)$. The final score in the LARS score ranges from 0 to 42; a score below 20 points indicates an absence of LARS, 21-29 is interpreted as a minor LARS and 29 up to 42 as a major LARS.

\section{EORTC-C30 and C29}

The EORTC-C30 measures the QoL regarding a global health status and contains five functional and nine symptoms scales. Depending on the responses by patients, according to the EORTC

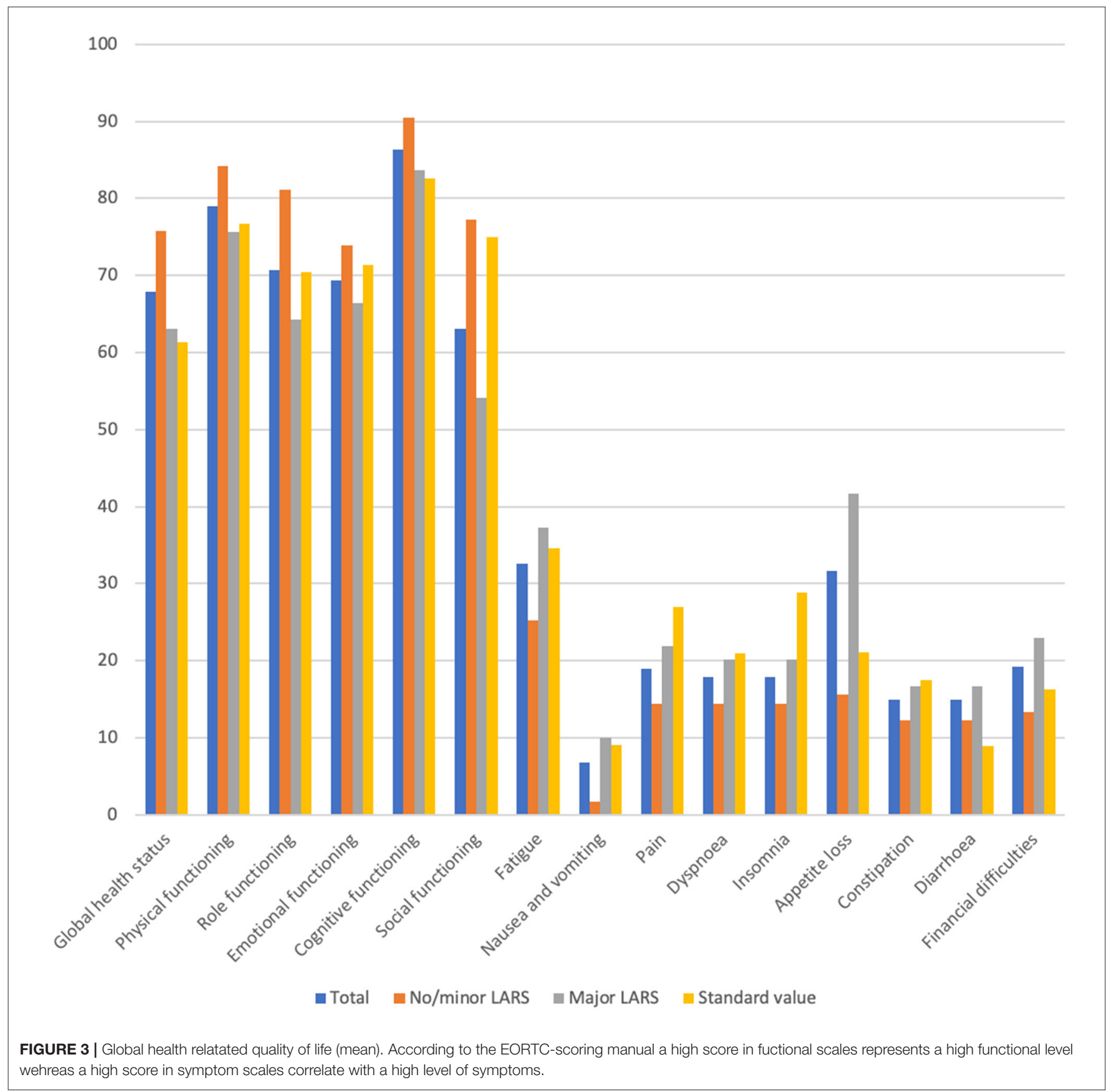


manual, a score ranging from 0 to 100 is calculated (17). Pursuant to the EORTC scoring system, a high score for the function or global health status indicates a better HRQOL; whereas a higher symptom scale means a greater burden by the scored symptom (17).

This tool was specified by an organ related module for colorectal malignancy with further 38 specific questions (QLQCR29) (32). The EORTC-C29 questionnaire was also scored following the published EORTC scoring manual (32). Some questions are focused on stoma-related issues which are excluded for non-stoma-patients. All data are compared to evaluate reference values (33). Also this combined EORTC-questionnaire with 68 items in total is very long, it provides a conclusive impression about the individual quality of life including organspecific complications.

\section{Statistical Analysis}

All data was analyzed using SPSS 25.0 (IBM Corp, Ammonk, NY). The Chi-Square-test was used to compare categorical

TABLE 2 | Patient characteristics according to the global health status.

\begin{tabular}{|c|c|c|c|}
\hline & $\begin{array}{l}\text { Global health } \\
\text { status > } 65 \\
\quad(n=51)\end{array}$ & $\begin{array}{l}\text { Global health } \\
\text { status }<65 \\
(n=27)\end{array}$ & $p$-value \\
\hline Age [years] (mean \pm SD) & $65.06 \pm 11.10$ & $66.74 \pm 14.32$ & $\mathrm{NS}^{\mathrm{c}}$ \\
\hline $\mathrm{BMI}($ mean $\pm \mathrm{SD})$ & $28.21 \pm 10.23$ & $26.18 \pm 5.50$ & $\mathrm{NS}^{\mathrm{c}}$ \\
\hline \multicolumn{4}{|l|}{ Sex, n (\%) } \\
\hline Male & $30(58.8)$ & $15(55.6)$ & $N S^{b}$ \\
\hline Female & $21(41.2)$ & $12(44.4)$ & $N S^{b}$ \\
\hline \multicolumn{4}{|l|}{ ASA, n (\%) } \\
\hline 1 & $3(5.9)$ & $1(3.7)$ & $N S^{b}$ \\
\hline$\|$ & 35 (68.6) & $19(70.4)$ & $N S^{b}$ \\
\hline III & $13(25.5)$ & $7(25.9)$ & $N S^{b}$ \\
\hline \multicolumn{4}{|l|}{ Technique, n (\%) } \\
\hline Laparoscopic & $44(86.3)$ & $24(88.9)$ & $N S^{b}$ \\
\hline Open & $5(9.8)$ & $3(11.1)$ & $N S^{b}$ \\
\hline Conversion & $2(3.9)$ & $0(0.0)$ & $N S^{b}$ \\
\hline $\begin{array}{l}\text { Length of surgery } \\
\text { [min] (mean } \pm \text { SD) }\end{array}$ & $254.04 \pm 74.65$ & $280.96 \pm 86.51$ & $N S^{c}$ \\
\hline $\begin{array}{l}\text { Time to follow-up } \\
\text { [month] (mean } \pm \text { SD) }\end{array}$ & $19.02 \pm 17.18$ & $20.41 \pm 16.52$ & $N S^{c}$ \\
\hline Major LARS, n (\%) & 28 (54.9) & $20(74.1)$ & $N S^{b}$ \\
\hline Major complication (DC > 3b), n (\%) & $2(3.9)$ & $2(7.4)$ & $N S^{b}$ \\
\hline \multicolumn{4}{|l|}{ Dignity, n (\%) } \\
\hline Benign & $12(23.5)$ & $4(14.8)$ & $N S^{b}$ \\
\hline \multirow[t]{2}{*}{ Malignant } & $39(76.5)$ & $23(85.2)$ & $N S^{b}$ \\
\hline & $n=39$ & $n=23$ & \\
\hline $\mathrm{N}+(\%)^{\mathrm{a}}$ & $18(46.2)$ & $6(26.1)$ & $N S^{b}$ \\
\hline T3/4 (\%) & $19(48.7)$ & $10(43.5)$ & $N S^{b}$ \\
\hline $\mathrm{RO}(\%)^{\mathrm{a}}$ & 38 (97.4) & $23(100.0)$ & $N S^{b}$ \\
\hline
\end{tabular}

alncluding only cases with malignancy $(n=62)$.

${ }^{b}$ Fisher exact test.

${ }^{c}$ Man-Whitney-U-test. variables, and in case of $<25$ cases, the Fisher's exact test was used. In case of more than two groups, Kruskal-Wallis-test was performed. Continuous variables are presented as means and standard deviation as exemplified by the EORTC (17). For intergroup-evaluation, according to the EORTC and previous studies differences, were rated 5-10 as small difference, 10-20 moderate and more than 20 as a large difference (5). The Mann-Whitney $\mathrm{U}$-test was used for inter-group comparison. A $p$-value of $<0.05$ was defined statistically significant.

\section{RESULTS}

During the study timeframe, 1.987 colorectal surgical procedures were performed in our center. This included 153 patients with rectal resections and with primary anastomosis. Twelve patients (7.8\%) died during the follow-up-period and 12 patients $(7.8 \%)$ were lost to follow-up. The questionnaire including the written consent form was sent to all remaining 129 patients. Seventyeight returned the completed questionnaire (60.5\%). Overall, there were 45 male (57.7\%) and 33 females (42.3\%) with a mean age of $65.64 \pm 12.24$ years. As many as $20.5 \%$ of the surgical procedures were performed for benign rectal lesions such as extended diverticulitis or large polyp of the rectum and $79.5 \%$ procedures were performed for malignant rectal growths. In this study cohort, no AL was reported. At the same time, 67 patients $(85.9 \%)$ had an uneventfull recovery. Major complications requiring intervention under general anesthesia (Dindo-Clavien $>3 \mathrm{~b}$ ) were found in four patients $(5.1 \%)$. These included one stoma revision, one subcutaneous hematoma, one uretheral obstruction without injury of the urethera, and a case of splenic bleeding which was treated by splenectomy.

In our series, the majority $(87.2 \%$ were operated laparoscopically with no postoperative AL. The mean time to follow-up were 19.5 months. Major LARS was detected in 48 (61.5\%) patients. There was no significant correlation between the time to follow-up- and the rate of major LARS following the end-to-end rectal reconstruction. Patients' characteristics did not differ significantly according to no/minor LARS or major LARS are shown in Table 1. The duration of surgery was significantly longer in patients with major LARS (285.15 min $\pm 77.05 \mathrm{~min}$ ) than in patients without major LARS $(228.50 \mathrm{~min} \pm 71.37 \mathrm{~min}$, p-value 0.001).

Using the EORTC-C30 questionnaire, the mean global health status score in our study cohort was 67.95 points. This score differed significantly between the major LARS and no/minor LARS groups (63.02 vs. 75.83, $p=0.003)$. A significant difference was observed between these groups in terms of physical functioning $(p=0.031)$, role functioning $(\mathrm{p}=0.012)$, social functioning $(p=0.002)$, and nausea and vomiting ( $p$ $=0.017$ ) (Figure 3 ). There were no significant differences in patients' characteristics for a low or high global health status (Table 2).

Focusing on the EORTC-C29 questionnaire, we observed significant differences between no/minor LARS and major LARS groups for urinary frequency $(p=0.003)$, urinary incontinence $(p=0.007)$, buttock pain $(p<0.001)$, bloating 
100

90
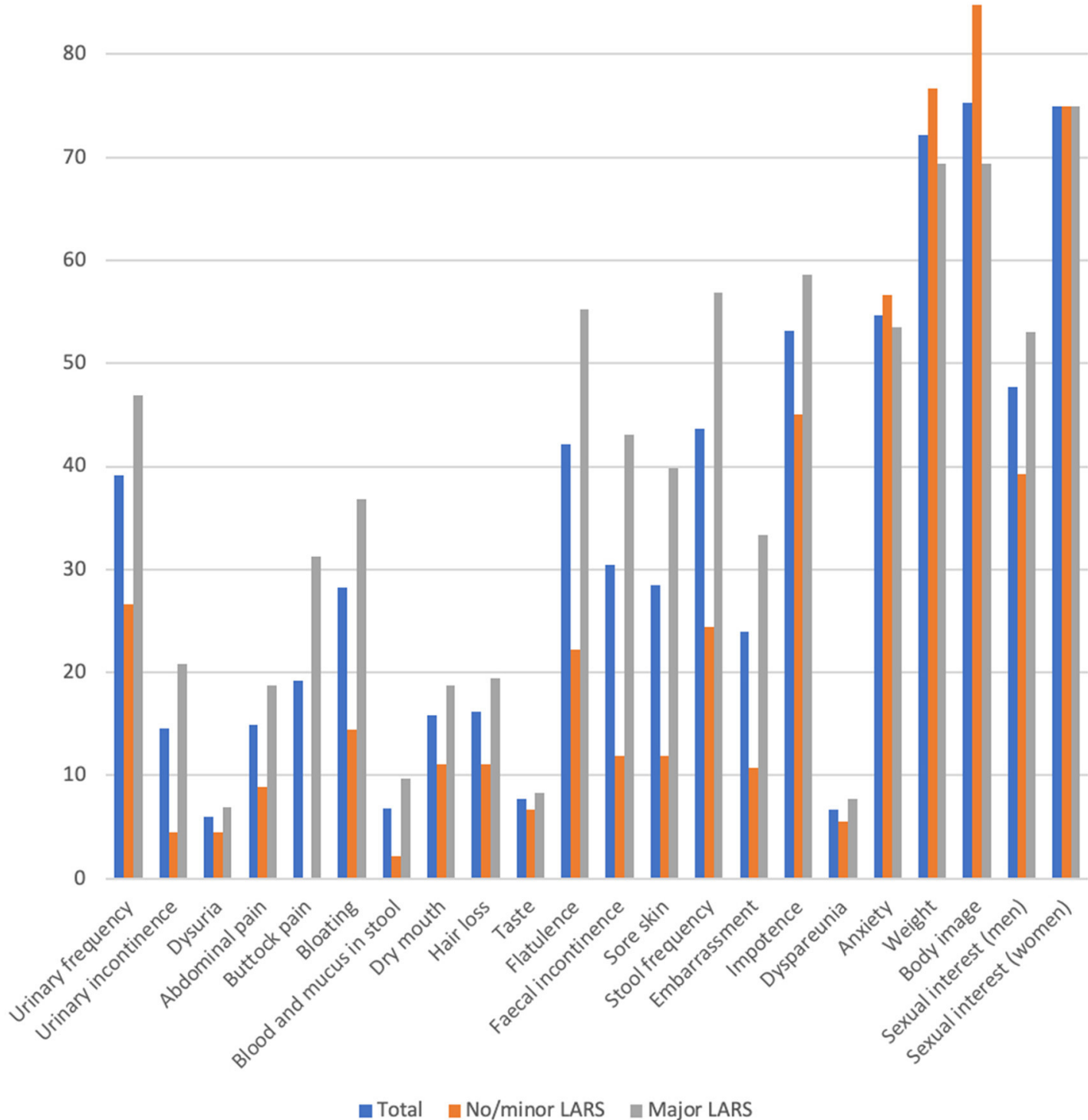

FIGURE 4 | Results of the QLQ-CR 29 questionnaire comparing no/minor with major LARS (mean). Scoring according to the EORTC-scoring manual a high score in fuctional scales represents a high functional level wehreas a high score in symptom scales correlate with a high level of symptoms.

$(p=0.006)$, blood and mucus in stool $(p=0.011)$. In addition, significant differences were found for flatulence $(p$ $<0.001)$, faecal incontinence $(p<0.001)$, stool frequency $(p<0.001)$ and sore skin $(p=0.003)$. Apart from the embarrassment $(p=0.008)$ and body image $(p=0.021)$, no further significant difference was reported for the sexual functioning (Figure 4).
The choice of the surgical approach (68 laparoscopic vs. 10 open) did not influence the mean LARS-score or the global health status. Furthermore, malignant nature of the rectal lesions had no significant impact on the postoperative mean global health status or the occurrence of a postoperative LARS (Table 3). The reported comorbidities such as cardiac or pulmonary diseases did not affect the postoperative LARS-score or the mean global 
health status. Regarding the Man-Whitney-U-test, the need of a life-long medication for any medical condition did not have a significant impact on the LARS $(p=0.906)$ or global health score $(p=0.812)$.

Using the Kruskal-Wallis-test, we did not find a significant change in the LARS-score according to the time to follow up $(p=0.676)$. Additionally, no significant change was noticed in the follow-up-period in the global health status with a mean of 68 points $(p=0.465)$ (Table 4$)$.

\section{DISCUSSION}

In our study, following a multimodal fail-safe perioperative protocol for rectal resections with end-to-end anastomosis and a temporary covering ileostomy, the patient-reported outcomes showed a high HRQOL with a global health status of $67.95 \pm 20.37$ points with no AL. The results signal the advantages of using a perioperative multimodal management protocol by adhering to surgical details in a structured fail-safe-protocol, thus reducing the postoperative $\mathrm{AL}$ rate with good functional outcomes after rectal resections and primary reconstruction.

Rectal resection causes the loss of specialized organ functions such as its reservoir function and the particularly the impairment of coordination between colonic movement, autonomic nerves and sphincter muscles. This complex interaction causes an increasing compartmentation following extended resection and deep anastomosis (34). This causes a rise in the LARS score following rectal dissection, resection and anastomosis, the surgical steps which explain the pathophysiology of LARS.

TABLE 3 | Global health status and LARS-Score according to potentia influencing factors.

\begin{tabular}{lccc}
\hline & Laparoscopic & Open/Conversion & p-value \\
\hline Global Health status (mean \pm SD) & $67.89 \pm 19.96$ & $68.33 \pm 24.15$ & 0.712 \\
LARS-score (mean \pm SD) & $29.22 \pm 11.20$ & $24.30 \pm 14.06$ & 0.216 \\
\hline & Benign & Malignant & p-value \\
\hline Global Health status (mean \pm SD) & $63.02 \pm 23.95$ & $69.22 \pm 19.36$ & 0.600 \\
LARS-score (mean \pm SD) & $26.81 \pm 11.82$ & $29.05 \pm 11.62$ & 0.339 \\
\hline
\end{tabular}

Higher score in Global health status means better global health status. Man-Whitney-U-test.
This has led to the development of different techniques for rectal reconstruction to construct a new reservoir using a pouch or a coloplasty. The advantages of the rectal reconstruction using a J-pouch have been shown by different international multicenter trials (35). Until today, clinical trials have not provided a concrete advantage of the J-pouch compared to other non-straight reconstruction modes $(36,37)$. On the same note, the study by Kupsch et al. did not report any notable difference between different non-straight-reconstruction modes for functional outcomes (38). Consequently, the recommendation by the German Guidelines for colorectal cancer is only a non-straight anastomosis, wherever achievable (26). A great majority of studies have shown better clinical outcomes within the first months after the rectal resection for non-straight anastomosis, but a reduced advantage in long-term follow-up $(36,39)$. Rybakov et al. compared straight vs. side-to-end anastomosis describing less bowel movements as the only benefit after 6 months (22). On the other hand, Lazorthes et al. showed functional improvements after rectal resections for 24 months (23). The long-term outcomes of a non-straight reconstruction after rectal resection remain unclear. According to our analysis, we could not find a major change of global health status or LARS-score over the follow-up-period (Table 4).

A relatively new surgical technique for rectal resection is the transanal total mesorectal excision (TaTME), which has shown comparable postoperative outcomes in the initial phase (40). Further studies showed more inconsistent results regarding this technique. De Simone et al. have described acceptable functional outcomes during the short-term-follow-up (41), while other studies have reported high rates of complications especially AL rates. These findings have provided an impetus to abandon the TaTME approach (42). Additionally, Bianco et al. have recently published a new technique for the rectal resection with an adopted pull-through anastomosis (43). This technique has demonstrated a comparable mean LARS-score after 12 and 36 months and a comparatively low leakage rate.

As a part of the multimodal fail-safe-concept used in our institution, a reconstruction using pouch or coloplasty or even side-to-end-anastomosis was not used to reduce the rate of AL. The studies evaluating the functional improvements by a pouch, side-to-end-reconstruction or coloplasty showed a relative high rate of AL. The meta-analysis presented by Hüttner et al. showed no significant differences in AL rate according to different reconstruction techniques with an $\mathrm{AL}$ rate ranging from $3.6 \%$ in J-pouch to $9.9 \%$ in another J-pouch-group (36). The AL rate for straight reconstruction was as high as $7.7 \%$. In

TABLE 4 | LARS and global health status according to time to follow-up (months).

\begin{tabular}{|c|c|c|c|c|c|c|c|c|}
\hline & $\begin{array}{c}0-12 \\
N=39\end{array}$ & $\begin{array}{c}13-24 \\
N=18\end{array}$ & $\begin{array}{l}25-36 \\
N=7\end{array}$ & $\begin{array}{l}37-48 \\
N=6\end{array}$ & $\begin{array}{l}49-60 \\
N=7\end{array}$ & $\begin{array}{c}>60 \\
N=1\end{array}$ & $\begin{array}{c}\text { Total } \\
N=78\end{array}$ & $p$-value \\
\hline LARS (mean \pm SD) & $29.97 \pm 9.94$ & $30.61 \pm 10.02$ & $22.29 \pm 16.93$ & $24.67 \pm 12.52$ & $24.14 \pm 16.87$ & 37.00 & $28.59 \pm 11.62$ & $0.676^{a}$ \\
\hline Global Health status (mean \pm SD) & $69,87 \pm 17.59$ & $59.72 \pm 21.82$ & $76.19 \pm 20.65$ & $69.44 \pm 19.48$ & $69.05 \pm 31.07$ & 66.67 & $67.95 \pm 20.37$ & $0.465^{a}$ \\
\hline
\end{tabular}

${ }^{a}$ Kruskal-Wallis-Test. 
our cohort, 0\% AL was recorded. Further refinements such as laparoscopic (18) or robotic surgery (44), pelvic intraoperative neuromonitoring (45), transanal total mesorectal excision (31) or fluorescence-guided imaging (46) may reduce the rates of $\mathrm{AL}$ and enhance the functional outcomes with or without a new reservoir made by pouch, side-to-end anastomosis or coloplasty.

\section{HRQOL}

In our study, a major LARS occurred in $61.5 \%$ patients. This rate is in line with the internationally published data ranging from $41 \%$ (16) up to $52 \%$, as reported by Juul et al. (13).

In 2019, Kupsch et al. compared the correlation between LARS and the QoL using the EORTC-questionnaires (5). In their study, the investigators found a reduced global quality of life, according to the EORTC-C30 questionnaire, in the group of patients with major LARS. This is also seen in our study whereas the measured global health status in patients with major LARS was higher $[63 \pm 20$ vs. $56 \pm 19$ Kupsch et al. (5)].

\section{Limitations}

As this is a retrospective analysis on the basis of a prospective database, no longitudinal comparison is achievable. Our data presents a median follow up of 1 year. A more longitudinal study design could establish the efficacy of the multi-modal failsafe-protocol with substantial impact. Due to the small number of the answered questionnaires, the size of our study is small. Additionally, there are no internal or external control-groups with non-straight anastomosis, so an inter-group or pairwise comparison was not possible.

This study includes postoperative patients after surgery for benign and malignant rectal lesions. Even if there was no significant difference in the global QoL between both groups, this is a major study limitation, as the QoL and LARS could be influenced by neoadjuvant or adjuvant treatment, even if the surgery is performed following oncological criteria.

\section{REFERENCES}

1. Guraya SY. Pattern, stage, and time of recurrent colorectal cancer after curative surgery. Clin Colorectal Cancer. (2019) 18:e223-8. doi: 10.1016/j.clcc.2019.01.003

2. Baidoun F, Elshiwy K, Elkeraie Y, Merjaneh Z, Khoudari G, Sarmini MT, et al. Colorectal cancer epidemiology: recent trends and impact on outcomes. Curr Drug Targets. (2021) 22:998-1009. doi: 10.2174/13894501219992011171 15717

3. Sandberg S, Asplund D, Bisgaard T, Bock D, González E, Karlsson L, et al. Low anterior resection syndrome in a Scandinavian population of patients with rectal cancer: a longitudinal follow-up within the QoLiRECT study. Colorectal Dis. (2020) 22:1367-78. doi: 10.1111/codi.15095

4. Rausa E, Bianco F, Kelly ME, Aiolfi A, Petrelli F, Bonitta G, et al. Systemic review and network meta-analysis comparing minimal surgical techniques for rectal cancer: quality of total mesorectum excision, pathological, surgical, and oncological outcomes. J Surg Oncol. (2019) 119:987-98. doi: 10.1002/jso.25410

5. Kupsch J, Kuhn M, Matzel KE, Zimmer J, Radulova-Mauersberger O, Sims A, et al. To what extent is the low anterior resection syndrome (LARS) associated with quality of life as measured using the EORTC C30 and CR38 quality of life questionnaires? Int J Colorectal Dis. (2019) 34:747-62. doi: 10.1007/s00384-019-03249-7

\section{CONCLUSION}

Our study eludes that the functional outcomes following rectal resections with straight anastomosis are not worse than reported by reconstruction with J-pouch-, side-to-end anastomosis or coloplasty, even within the first 12 month of surgery. Despite our small study group, we emphasis that we did not record even a single AL following rectal resections and primary end-to-end anastomosis with temporary covering ileostomy. In conclusion, the straight anastomosis after rectal resection is an achievable procedure with a good functional outcome and a reduced leakage rate following the multimodal fail-safe-protocol.

\section{DATA AVAILABILITY STATEMENT}

The raw data supporting the conclusions of this article are available on request from the corresponding author.

\section{ETHICS STATEMENT}

The studies involving human participants were reviewed and approved by Ethics Committee Medical Association SchleswigHolstein Bismarckallee 8-12 23795 Bad Segeberg Germany. The patients/participants provided their written informed consent to participate in this study.

\section{AUTHOR CONTRIBUTIONS}

$\mathrm{JH}$ and $\mathrm{VB}$ collected and analyzed the data and drafted the manuscript. SK and $\mathrm{HH}$ drafted and reviewed the manuscript and made an impact on discussion. TS reviewed the manuscript and supervised the study. SG performed a scientific enrichment, linguistics manuscript drafting, and statistical analysis. All authors read and approved the final manuscript.

6. Williams NS, Johnston D. The quality of life after rectal excision for low rectal cancer. Br J Surg. (1983) 70:460-2. doi: 10.1002/bjs.1800700805

7. EORTC. European Organisation for Research and Treatment of Cancer: EORTC. Available online at: http://www.eortc.org/ (accessed November 1, 2017).

8. Rullier E, Laurent C, Garrelon JL, Michel P, Saric J, Parneix M. Risk factors for anastomotic leakage after resection of rectal cancer. Br J Surg. (1998) 85:355-8. doi: 10.1046/j.1365-2168.1998.00615.x

9. Croese AD, Lonie JM, Trollope AF, Vangaveti VN, Ho Y-H, A. meta-analysis of the prevalence of Low Anterior Resection Syndrome and systematic review of risk factors. Int J Surg. (2018) 56:234-41. doi: 10.1016/j.ijsu.2018.06.031

10. Turco LC, Tortorella L, Tuscano A, Palumbo MA, Fagotti A, Uccella S, et al. Surgery-related complications and long-term functional morbidity after segmental colo-rectal resection for deep infiltrating endometriosis (ENDO-RESECT morb). Arch Gynecol Obstet. (2020) 302:983-93. doi: 10.1007/s00404-020-05694-0

11. Bryant CLC, Lunniss PJ, Knowles CH, Thaha MA, Chan CLH. Anterior resection syndrome. Lancet Oncol. (2012) 13:e403-8. doi: 10.1016/S1470-2045(12)70236-X

12. Temple LK, Bacik J, Savatta SG, Gottesman L, Paty PB, Weiser $\mathrm{MR}$, et al. The development of a validated instrument to evaluate bowel function after sphincter-preserving surgery for rectal cancer. 
Dis Colon Rectum. (2005) 48:1353-65. doi: 10.1007/s10350-004-0 942-z

13. Juul T, Ahlberg M, Biondo S, Emmertsen KJ, Espin E, Jimenez LM, et al. International validation of the low anterior resection syndrome score. Ann Surg. (2014) 259:728-34. doi: 10.1097/SLA.0b013e3182 $8 \mathrm{fac} 0 \mathrm{~b}$

14. Juul T, Battersby NJ, Christensen P, Janjua AZ, Branagan G, Laurberg S, et al. Validation of the English translation of the low anterior resection syndrome score. Colorectal Dis. (2015) 17:908-16. doi: 10.1111/codi.12952

15. Bittorf B, Matzel KE. Der LARS-Score zur Evaluation des anterioren Resektionssyndroms. Coloproctology. (2015) 37:262-5. doi: 10.1007/s00053-015-0028-5

16. Emmertsen KJ, Laurberg S. Low anterior resection syndrome score: development and validation of a symptom-based scoring system for bowel dysfunction after low anterior resection for rectal cancer. Ann Surg. (2012) 255:922-8. doi: 10.1097/SLA.0b013e31824f1c21

17. Aaronson NK, Ahmedzai S, Bergman B, Bullinger M, Cull A, Duez NJ, et al. The European Organization for Research and Treatment of Cancer QLQ-C30: a quality-of-life instrument for use in international clinical trials in oncology. J Natl Cancer Inst. (1993) 85:365-76. doi: 10.1093/jnci/85.5.365

18. Runkel N, Reiser H. Nerve-oriented mesorectal excision (NOME): autonomic nerves as landmarks for laparoscopic rectal resection. Int J Colorectal Dis. (2013) 28:1367-75. doi: 10.1007/s00384-013-1705-x

19. Kinugasa Y, Arakawa T, Murakami G, Fujimiya M, Sugihara K. Nerve supply to the internal anal sphincter differs from that to the distal rectum: an immunohistochemical study of cadavers. Int J Colorectal Dis. (2014) 29:42936. doi: 10.1007/s00384-013-1811-9

20. Stelzner S, Wedel T. Anatomic principles of nerve-sparing rectal surgery. Coloproctology. (2015) 37:240-7. doi: 10.1007/s00053-015-0030-y

21. Stelzner S, Böttner M, Kupsch J, Kneist W, Quirke P, West NP, et al. Internal anal sphincter nerves - a macroanatomical and microscopic description of the extrinsic autonomic nerve supply of the internal anal sphincter. Color Dis. (2018) 20:O7-O16. doi: 10.1111/codi.13942

22. Rybakov EG, Pikunov DY, Fomenko OY, Chernyshov S V, Shelygin YA. Sideto-end vs. straight stapled colorectal anastomosis after low anterior resection: results of randomized clinical trial. Int J Colorectal Dis. (2016) 31:1419-26. doi: $10.1007 / \mathrm{s} 00384-016-2608-4$

23. Lazorthes F, Fages P, Chiotasso P, Lemozy J, Bloom E. Resection of the rectum with construction of a colonic reservoir and colo-anal anastomosis for carcinoma of the rectum. Br J Surg. (1986) 73:136-8. doi: 10.1002/bjs. 1800730222

24. Fazio VW, Mantyh CR, Hull TL. Colonic "coloplasty": novel technique to enhance low colorectal or coloanal anastomosis. Dis Colon Rectum. (2000) 43:1448-50. doi: 10.1007/BF02236645

25. Huber FT, Herter B, Siewert JR. Colonic pouch vs. side-to-end anastomosis in low anterior resection. Dis Colon Rectum. (1999) 42:896-902. doi: $10.1007 / \mathrm{BF} 02237098$

26. Deutsche Krebsgesellschaft, Deutsche Krebshilfe, AWMF. German Guideline for Colorectal Cancer. (2019). Available online at: https://www.awmf.org/ uploads/tx_szleitlinien/021-007OLl_S3_Kolorektales-Karzinom-KRK_201901.pdf (accessed September 29, 2020).

27. Trenti L, Galvez A, Biondo S, Solis A, Vallribera-Valls F, Espin-Basany E, et al. Quality of life and anterior resection syndrome after surgery for mid to low rectal cancer: A cross-sectional study. Eur J Surg Oncol. (2018) 44:1031-9. doi: 10.1016/j.ejso.2018.03.025

28. Battersby NJ, Juul T, Christensen P, Janjua AZ, Branagan G, Emmertsen KJ, et al. Predicting the risk of bowel-related quality-of-life impairment after restorative resection for rectal cancer: a multicenter cross-sectional study. Dis Colon Rectum. (2016) 59:270-80. doi: 10.1097/DCR.0000000000000552

29. von Elm E, Altman DG, Egger M, Pocock SJ, Gøtzsche PC, Vandenbroucke JP. STROBE Initiative. The Strengthening the Reporting of Observational Studies in Epidemiology (STROBE) statement: guidelines for reporting observational studie. Epidemiology. (2007) 18:800-4. doi: 10.1097/EDE.0b013e3181577654

30. Dindo D, Demartines N, Clavien P-A. Classification of surgical complications: a new proposal with evaluation in a cohort of 6336 patients and results of a survey. Ann Surg. (2004) 240:205-13. doi: 10.1097/01.sla.0000133083.54934.ae
31. Veltcamp Helbach M, Koedam TWA, Knol JJ, Velthuis S, Bonjer HJ, Tuynman JB, et al. Quality of life after rectal cancer surgery: differences between laparoscopic and transanal total mesorectal excision. Surg Endosc. (2019) 33:79-87. doi: 10.1007/s00464-018-6276-z

32. Whistance RN, Conroy T, Chie W, Costantini A, Sezer O, Koller M, et al. Clinical and psychometric validation of the EORTC QLQ-CR29 questionnaire module to assess health-related quality of life in patients with colorectal cancer. Eur J Cancer. (2009) 45:3017-26. doi: 10.1016/j.ejca.2009.08.014

33. Scott NW, Fayers PM, Aaronson NK, Bottomley A, De Graeff A, Groenvold $\mathrm{M}$, et al. EORTC QLQ-C30 Reference Values This manual presents reference data for the QLQ-C30 based upon data provided by EORTC Quality of Life Group Members and other users of the QLQ-C30 Sprangers on behalf of the EORTC Quality of Life Group EORTC Quality of Life Group. (2008). Available online at: https://www.eortc.org/app/uploads/sites/2/2018/ 02/reference_values_manual2008.pdf (accessed August 14, 2020).

34. Matzel KE, Stadelmaier U, Hohenberger W, Muehldorfer S. Continence after colorectal reconstruction following resection: impact of level of anastomosis. Int J Colorectal Dis. (1997) 12:82-7. doi: 10.1007/s003840050086

35. Fazio VW, Zutshi M, Remzi FH, Parc Y, Ruppert R, Fürst A, et al. A randomized multicenter trial to compare long-term functional outcome, quality of life, and complications of surgical procedures for low rectal cancers. Ann Surg. (2007) 246:481-90. doi: 10.1097/SLA.0b013e3181485617

36. Hüttner FJ, Tenckhoff S, Jensen K, Uhlmann L, Kulu Y, Büchler MW, et al. Meta-analysis of reconstruction techniques after low anterior resection for rectal cancer. Br J Surg. (2015) 102:735-45. doi: 10.1002/bjs.9782

37. Doeksen A, Bakx R, Vincent A, van Tets WF, Sprangers MAG, Gerhards MF, et al. J-pouch vs side-to-end coloanal anastomosis after preoperative radiotherapy and total mesorectal excision for rectal cancer: a multicentre randomized trial. Color Dis. (2012) 14:705-13. doi: 10.1111/j.1463-1318.2011.02725.x

38. Kupsch J, Jackisch T, Matzel KE, Zimmer J, Schreiber A, Sims A, et al. Outcome of bowel function following anterior resection for rectal cancer-an analysis using the low anterior resection syndrome (LARS) score. Int J Colorectal Dis. (2018) 33:787-98. doi: 10.1007/s00384-018-3006-x

39. Ribi K, Marti WR, Bernhard J, Grieder F, Graf M, Gloor B, et al. Quality of life after total mesorectal excision and rectal replacement: comparing side-to-end, colon $\mathrm{j}$-pouch and straight colorectal reconstruction in a randomized, phase III trial (SAKK 40/04). Ann Surg Oncol. (2019) 26:3568-76. doi: 10.1245/s10434-019-07525-2

40. European Society of Coloproctology (ESCP) collaborating group. An international multicentre prospective audit of elective rectal cancer surgery; operative approach versus outcome, including transanal total mesorectal excision (TaTME). Colorectal Dis. (2018) 20(Suppl. 6):33-46. doi: 10.1111/codi. 14376

41. De Simone V, Persiani R, Biondi A, Litta F, Parello A, Campennì P, et al. One-year evaluation of anorectal functionality and quality of life in patients affected by mid-to-low rectal cancer treated with transanal total mesorectal excision. Updates Surg. (2021) 73:157-64. doi: 10.1007/s13304-020-0 0919-y

42. Wasmuth HH, Faerden AE, Myklebust TÅ, Pfeffer F, Norderval S, Riis $\mathrm{R}$, et al. Transanal total mesorectal excision for rectal cancer has been suspended in Norway. Br J Surg. (2020) 107:121-30. doi: 10.1002/bjs. 11459

43. Bianco F, Incollingo P, Falato A, De Franciscis S, Belli A, Carbone F, et al. Short stump and high anastomosis pull-through (SHiP) procedure for delayed coloanal anastomosis with no protective stoma for low rectal cancer. Updates Surg. (2021) 73:495-502. doi: 10.1007/s13304-021-01022-6

44. Luca F, Valvo M, Guerra-Cogorno M, Simo D, Blesa-Sierra E, Biffi R, et al. Functional results of robotic total intersphincteric resection with hand-sewn coloanal anastomosis. Eur J Surg Oncol. (2016) 42:841-7. doi: 10.1016/j.ejso.2016.03.007

45. Kneist W, Rink AD, Kauff DW, Konerding MA, Lang H. Topography of the extrinsic internal anal sphincter nerve supply during laparoscopic-assisted TAMIS TME: five key zones of risk from the surgeons' view. Int $J$ Colorectal Dis. (2015) 30:71-8. doi: 10.1007/s00384-014-2026-4

46. Blanco-Colino R, Espin-Basany E. Intraoperative use of ICG fluorescence imaging to reduce the risk of anastomotic leakage in colorectal surgery: 
a systematic review and meta-analysis. Tech Coloproctol. (2018) 22:15-23. doi: 10.1007/s10151-017-1731-8

Conflict of Interest: The authors declare that the research was conducted in the absence of any commercial or financial relationships that could be construed as a potential conflict of interest.

Publisher's Note: All claims expressed in this article are solely those of the authors and do not necessarily represent those of their affiliated organizations, or those of the publisher, the editors and the reviewers. Any product that may be evaluated in this article, or claim that may be made by its manufacturer, is not guaranteed or endorsed by the publisher.

Copyright $\odot 2022$ Herzberg, Khadem, Begemann, Strate, Honarpisheh and Guraya. This is an open-access article distributed under the terms of the Creative Commons Attribution License (CC BY). The use, distribution or reproduction in other forums is permitted, provided the original author(s) and the copyright owner(s) are credited and that the original publication in this journal is cited, in accordance with accepted academic practice. No use, distribution or reproduction is permitted which does not comply with these terms. 